\title{
Graph Link Invariants of Isolated Singularities of Holomorphic Vector Fields in $\mathrm{C}^{2}$ II
}

\author{
Nobuatsu OKA \\ (Communicated by K. Akao)
}

\section{Introduction.}

The contents of this note consist of two parts. One is a generalization of our results in the previous paper [8]. The other is to present a way of computing the multiplicity of the splice diagram induced by a holomorphic vector fields on $\mathbf{C}^{2}$ announced in [8].

In $\$ 2$, we study some topological invariants around the isolated singularity of a holomorphic vector field. The graph called a splice diagram was constructed from a given generalized curve in [8]. We show by using the splice diagram that the Thurston norm and the one-variable Alexander polynomial are topological invariants around the singularity of vector fields. the splice diagram can be also defined from a holomorphic vector field on $\mathbf{C}^{2}$. We show that the Thurston norm and the one-variable Alexander polynomial are also topological invariants for holomorphic vector fields on $\mathbf{C}^{2}$.

First we recall the concept of multiplicity of multi-graph link. This link is represented by a splice diagram with arrowhead vertices. Each arrowhead vertex in a diagram corresponds to a component of a given multi-graph link. All arrowhead vertices have an integer called a vertex weight which corresponds to the multiplicity of a link component of a graph link. We refer to [5], [6], [7] and [8] for further interpretations on a graph link, splice diagram, and arrowhead vertex of splice diagram. The multiplicity of a link is defined as follows. Let $\Sigma$ be a homology 3-sphere and $(\Sigma, K)$ be an unoriented link. Let $L=\left(\Sigma, S_{1} \cup \cdots \cup S_{n}\right)$ be a link obtained by orienting $(\Sigma, K)$. A multi-link on $(\Sigma, K)$ means a link $L$ together with an integer $m_{i}$ associated with each component $S_{i}$. A component $-S_{i}\left(S_{i}\right.$ with reversed orientation) with an integer $-m_{i}$ is the same as $S_{i}$ with an integer $m_{i}$. Hence a link is simply a multi-link with an integer \pm 1 . We call the integer $m_{i}$ multiplicity of a component $S_{i}$, and the set of integers $\left(m_{1}, \cdots, m_{n}\right)$ multiplicities of a multi-graph link.

A multi-link on $(\Sigma, K)$ determines an integral cohomology class $m \in H^{1}(\Sigma-K)$, and the class $m$ is evaluated on a 1-cycle $S$ by the linking number:

Received September 24, 1993

Revised January 20, 1995 


$$
m(S)=\operatorname{Link}\left(m_{1} S_{1}+\cdots+m_{n} S_{n}, S\right)=\sum m_{i} \operatorname{Link}\left(S_{i}, S\right) .
$$

It follows from the Alexander duality that $n$ linear forms $\operatorname{Link}\left(S_{i},-\right) \in H^{1}(\Sigma-K)$ are a basis of $H^{1}(\Sigma-K)$. We identify this basis with $Z^{n}$, and a cohomology class $m$ with $\left(m_{1}, \cdots, m_{n}\right)$. From the view points of geometric topology the multiplicity of a component of the link means a positive integer which is summed up all over the winding number of several paralell cable knots around a component of the link. Here, the cable knots appear as the intersection between the boundary of the tubular neighborhood of a component of the given multi-graph link and its Seifert surface. For the details see [5].

Next, let us observe the multiplicities of the graph multi-link induced from a plane curve $f=0$. A Milnor fibration is defined for this curve. This fibration is a foliation induced by a Hamiltonian system defined by the next equation around the origin $o \in \mathbf{C}^{2}$;

$$
\dot{x}=-\frac{\partial f}{\partial y}, \quad \dot{y}=\frac{\partial f}{\partial x}, \quad(x, y) \in \mathbf{C}^{2} .
$$

Hence the multi-link is defined by the intersections between the separatrices of this Hamiltonian system and a small $S^{3}$ around the origin. The cable knots appear as the intersections between a leaf near the separatrix of this fibration and a small $S^{3}$. This leaf is a regular fiber of the Milnor fibration and is a Seifert surface of the multi-link. Thus the multiplicity of this link is equal to an integer which is summed up all over the winding number of several parallel cable knots around a component of this link.

In [8] the intersection between separatrices of a vector field and a boundary of a plumbed manifold was considered as a multi-graph link. Here a boundary of a plumbed manifold is a 3-sphere and coincides with a boundary of a certain neighborhood of the singularity of the vector field. Since separatrices of vector fields (integral curves of the vector fields passing through the singularity) are analytic curves (plane curves), the multiplicities of graph links defined in [8] are considered as the multiplicities of a graph link induced by separatrices of a certain Hamiltonian system.

To obtain the multiplicity we must calculate the integral curves (i.e., the separatrices) of a given vector field. However, we cannot usually get the concrete separatrices. Hence, as in $\S 3$, we consider the linear holonomy of the vector field around certain simple singularities appearing in the divisor of the final stage of blow-up processes. Foliations induced by a given vector field are not always Milnor fibrations. However we can define the type of the cable knot (see §3) for some vector fields. Here, this cable knot is also the intersection between the boundary of the small neighborhood of a component of the link and leaves near the separatrix. The components of the link define the intersections between separatrices and a boundary of a certain neighborhood of the singularity of our vector field like the case of Milnor fibrations. Hence we define here our multiplicity of link components by the winding number of the cable knot around the component of this link. The above cable knot is a connected component of the intersection between the Seifert surface of this multi-link and the boundary of the tubular neighborhood of 
the component of this link. So this multiplicity is an extension of the ordinary multiplicity of links defined in [8]. Notice that the above cable knot may not be all of the intersection between the Seifert surface and the boundary of the tubular neighborhood of a component of the link.

\section{Topological invariants of the general holomorphic vector fields with an isolated singularity $o$ in $\mathbf{C}^{2}$.}

We have the same results as Theorem A and Theorem B in [8] for the holomorphic vector fields with an isolated singularity $o$ defined in $\mathbf{C}^{2}$. We will prove this fact in this section. For the preparations we need several definitions and theorems.

Definition 1 ([2]). Let $Z$ be an analytic vector field written as $\dot{x}=x^{n} P(x)+$ $y Q(x, y), \dot{y}=y R(x, y)$, where $P(0) \neq 0$. Suppose that the equation $y=0$ defines a smooth invariant analytic line $S$ which contains the singularity $q$ of holomorphic foliation $F$ defined by $Z$. The integer $n \in \mathbf{N}$ is called the multiplicity of $F$ in $q \in S$ along $S$, and is denoted by $\mu_{F}(q, S)$.

It is clear that this multiplicity has no relation to the multiplicity of a multi-graph link.

Definition $2([2])$. The weight $\rho(P)$ of a projective line $P$ which appears in the process of desingularization is defined as

(1) 1 if $P$ appears immediately after exploding $o \in \mathbf{C}^{2}$,

(2) the sum of the weights of the projective lines meeting at the singularity with $P$ which is created by performing blowing-up operations at the singularity.

$\rho(P)$ is equal to the absolute value of the Chern number of the line bundle over the projective line $P$. So it is equal to the absolute value of the vertex weight of a vertex corresponding to the projective line $P$ defined as above.

We consider the desingularization $\left(U_{Z}, \pi, P_{Z}, F_{Z}\right)$ of a vector field $Z$. Let $p \in P$ be a singularity of $F_{Z}$ and put

$$
\begin{aligned}
& \phi(p, P)=\mu_{F_{z}}(p, P) \quad \text { if } \quad p \in P \text { is not a corner . } \\
& \mu_{F_{Z}}(p, P)-1 \quad \text { if } \quad p \in P \text { is a corner . }
\end{aligned}
$$

To prove Proposition 1 stated below, we need the following theorem proved in [2].

THEOREM 1 ([2]). The algebraic multiplicity $v=v_{Z}$ of $Z$ is given by $v+1=$ $\Sigma \rho(P) \phi(p, P)$, where the summation runs through the singularities of $F_{Z}$.

Proposition 1. Suppose that $Z$ and $Z^{\prime}$ are holomorphic vector fields having an isolated singularity o in $\mathbf{C}^{2}$. If there exists a topological equivalence between $Z$ and $Z^{\prime}$, then the minimal plumbing diagram defined by $Z$ is coincident with the one defined by $Z^{\prime}$.

Proof. Let $S$ and $S^{\prime}$ be separatrices of $Z$ and $Z^{\prime}$ respectively. Since there exists 
a topological equivalence between $Z$ and $Z^{\prime}$, both of the separatrices $S^{\prime}$ and $S$ are desingularized simultaneously by the blowing up processes of several times. Let $k$ denote the number of the blowing-up operations for the desingularization of separatrices $S$ and $S^{\prime}$. Let $Z^{(k)}$ and $Z^{\prime(k)}$ be vector fields induced from $Z$ and $Z^{\prime}$ by $k$ blowing-ups respectively.

Case (1). Singularities appearing on one component of divisors of $Z^{(k)}$ and $Z^{\prime(k)}$. Let $F_{Z}^{(k)}$ and $F_{Z^{\prime}}^{(k)}$ be foliations defined by the vector field $Z^{(k)}$ and $Z^{\prime(k)}$ respectively. If a leaf $l_{Z}$ of $F_{Z}^{(k)}$ transversely interesects to an invariant component $C P^{1}$, then there exists a leaf $l_{Z^{\prime}}$ of $F_{Z^{\prime}}^{(k)}$ corresponding to $l_{Z}$ under a leaf preserving homeomorphism. $l_{Z^{\prime}}$ also intersects transversely to an invariant component of the divisor. By using Lemma 1 in [2] we see that the algebraic multiplicities of the vector fields $Z^{(k)}$ and $Z^{\prime(k)}$ at the above intersection points are one. Now suppose that $\lambda_{1}^{(k)}$ and $\lambda_{2}^{(k)}\left(\lambda_{1}^{\prime(k)}\right.$ and $\lambda_{2}^{\prime(k)}$, resp.) are eigenvalues of $Z^{(k)}\left(Z^{\prime(k)}\right.$, resp.) at the above intersection point. It is easily checked that all eigenvalues $\lambda_{1}^{(k)}, \lambda_{2}^{(k)}, \lambda_{1}^{\prime(k)}$ and $\lambda_{2}^{\prime(k)}$ are not zero. We have no relations such as $m \lambda_{1}^{(k)}=n \lambda_{2}^{(k)}$ and $m^{\prime} \lambda_{1}^{\prime(k)}=n^{\prime} \lambda_{2}^{\prime(k)}$, where $m, n, m^{\prime}, n^{\prime} \in \mathbf{N}$. For, if there exist such relations, there exist infinitely many integral curves or exists only one integral curve passing through the above intersection point. However this fact contradicts that $Z^{(k)}$ and $Z^{\prime(k)}$ must have exactly two integral curves passing through the intersection point. Thus we conclude that the above intersection points are non-zero simple singularities of $Z^{(k)}$ and $Z^{\prime(k)}$.

Next we consider about a zero simple singularity occurring on an invariant component $C P^{1}$. All zero simple singularities have at least one invariant curve passing through the singularity (see [2]). If a zero simple singularity has more than two invariant curves, then it has exactly two, because all separatrices are already desingularized by performing several blowing-ups. These two invariant curves intersect transversely at the singularity. Thus one of them must be contained in the divisor. So we see that there exists one separatrix which intersects transversely to the divisor or there exists no such separatrix, i.e., all separatrices are contained in a divisor. If $Z^{(k)}$ has a separatrix of the former type of the zero simple singularity, then $Z^{\prime(k)}$ has also the corresponding separatrix by the leaf preserving homeomorphism between $Z^{(k)}$ and $Z^{\prime(k)}$. If $Z^{(k)}$ has separatrices of the latter type, then $Z^{\prime(k)}$ may not have corresponding separatrices. For the leaf preserving homeomorphism is not necessarily extendible to the divisor. However the separatrices of the latter type must be contained in a component of the divisor and so the separatrices are not represented as arrowhead lines in a plumbing diagram. Thus the existence of graph isomorphisms of plumbing diagrams for this case does not ensure the one to one correspondence between zero simple singularities of $Z^{(k)}$ and $Z^{\prime(k)}$

We can check that the existence of zero simple singularities in the divisor does not influence the isomorphisms between the plumbing diagrams $Z^{(k)}$ and $Z^{\prime(k)}$ in both cases. In fact, take a differential equation that $\dot{x}=\lambda_{1}^{(k)} x+\alpha y^{p}, \dot{y}=\lambda_{2}^{(k)} y^{p}$. Here, $(x, y)$ denotes a standard local coordinate around the origin in $\mathbf{C}^{2}, \lambda_{1}^{(k)} / \lambda_{2}^{(k)}=p \in \mathbf{N}$ and $\alpha \neq 0$. Assume that the singularity of the above type appears on an invariant component, that is, the 
topology of foliation near the singularity in $C P^{1}$ is equivalent to the topology of the foliation induced by the above equation around $o$. It does not ensure the one to one correspondence between the above type singularities of $Z^{(k)}$ and $Z^{\prime(k)}$ by a leaf preserving homeomorphism. Thus we need to perform more blowing-ups at the singularity for the desingularization of vector fields. It is clear that only one leaf passes through the singularity in this case, and the leaf must be contained in an invariant component. Thus no separatrices representing arrowhead lines in a plumbing diagram appear after performing blowing-ups at these singularities. By using blowing-down we can assume that there exist no such singularities on an invariant component. Any graph isomorphism between the plumbing diagrams of $Z^{(k)}$ and $Z^{\prime(k)}$ is not also ruined by the existence of singularities of these types. If the other types of singularities appear on the divisor, the separatrices of such singularities must be contained in the divisor, because all separatrices of $Z$ and $Z^{\prime}$ are already desingularized by performing several blowing-ups. Thus no separatrices represented by arrowhead lines in a plumbing diagram appear after performing blowing-ups at the singularities. Hence any graph isomorphism between the plumbing diagrams of $Z^{(k)}$ and $Z^{\prime(k)}$ is not ruined.

Case (2). Singularities in the intersection points (or the corners) between two components of the divisor. The case (2) is divided into the following two cases (see [2]).

(i) The corner is constructed by an invariant component and a critical component.

(ii) The corner is constructed by two invariant components.

The corner of type (i) (resp. type (ii)) in the divisor of $Z^{(k)}$ corresponds to the corner of type (i) (resp. (ii)) in the divisor of $Z^{\prime(k)}$ by a leaf preserving homeomorphism. There exist no separatrices which pass through the corner (or the intersection point) because separatrices $S$ and $S^{\prime}$ are already desingularized. In the case (i) the type of singularities appearing on the corners is zero simple singularity or the singularity defined by the equations $\dot{x}=\lambda_{1}^{(k)} x+\alpha y^{p}, \dot{y}=\lambda_{2}^{(k)} y$ as above. For the case of singularities defined by the above equations we need to perform the blowing-up at the corner for the desingularization of the vector field. However no new separatrices which transversely intersect to the divisor are created by the blowing-up. So, by using blow-down operations, the final resolution picture can be reduced to the one of which corner is zero simple singularity. Thus we assume that the singularities appearing on the corners are zero simple singularities for the case (i). In the case (ii) the intersection points are non-zero simple singularity.

Case (3). Singularities appearing in a critical component. All critical components of the divisor of $Z^{(k)}$ correspond to the critical components of the divisor of $Z^{\prime(k)}$ by a leaf preserving homeomorphism between $Z^{(k)}$ and $Z^{\prime(k)}$. If the vector field $Z^{(k)}$ and $Z^{\prime(k)}$ have a singularity on a critical component, then there exists only one leaf passing through each singularity. In this case, just one explosion is needed for each vector field $Z^{(k)}$ or $Z^{\prime(k)}$. After performing one explosion, both singularities become non-zero simple singularities. Hence the leaf preserving homeomorphism between $Z^{(k+1)}$ and $Z^{\prime(k+1)}$ induces the graph isomorphism between the minimal plumbing diagrams. Thus 
two minimal plumbing diagrams coincide.

COROLlaRY 3. Let $Z$ and $Z^{\prime}$ be vector fields as above, and assume that there exists a topological equivalence between $Z$ and $Z^{\prime}$. Then the minimal splice diagram of $Z$ is isomorphic to the minimal splice diagram of $Z^{\prime}$.

Now we obtain the same results as in Theorem A and Theorem B by Corollary 3 and the same arguments as in Theorems A and B in [8].

THEOREM C. Suppose that $Z$ and $Z^{\prime}$ are as above. If there exists a topological equivalence between $Z$ and $Z^{\prime}$, the minimal plumbing diagram $\Gamma_{Z}$ of $Z$ agrees with the plumbing diagram $\Gamma_{Z^{\prime}}$ of $Z^{\prime}$, and the pairs $\left(\Delta_{\Gamma_{Z}}, n_{Z}\right)$ and $\left(\Delta_{\Gamma_{Z^{\prime}}}, n_{Z^{\prime}}\right)$ coincide as in Theorem A.

TheOREM D. Suppose that $Z$ and $Z^{\prime}$ are as above. If there exists a topological equivalence between $Z$ and $Z^{\prime}$, the minimal splice diagram $T_{Z}$ of $Z$ agrees with the minimal splice diagram $T_{Z^{\prime}}$ of $Z^{\prime}$, and the norm $\left\|m_{Z}\right\|$ of $Z$ is equal to the norm $\left\|m_{Z^{\prime}}\right\|$.

Notice that, concerning general holomorphic vector fields defined on $\mathbf{C}^{2}$, even if given two vector fields are topologically equivalent to each other, the corresponding two plumbing diagrams may not be isomorphic. On the other hand, the corresponding two minimal plumbing diagrams are always isomorphic.

\section{On a holonomy of holomorphic vector field and a splice diagram.}

In this section we consider a relation between splice diagrams and certain holonomies of vector fields, and define the multiplicity of the graph link induced by a vector field as we announced in $\S 1$.

After desingularizing a given holomorphic vector field, we pay attention to non-zero simple singularities of which eigenvalues $\lambda_{1}$ and $\lambda_{2}$ satisfy the condition

$$
m_{1} \lambda_{1}+m_{2} \lambda_{2}=0 \quad \text { for some } m_{1}, m_{2} \in \mathbf{N} \text {. }
$$

We take sufficiently small 2-disks as fibers of the 2-disk bundle over every component of a divisor of the final resolution picture. For each singularity which satisfies the above condition, we take a small circle of which center is the singularity on the component $C P^{1}$ and on the integral curve passing through the singularity respectively. Construct a fence on each circle by using small 2-disks as in Figure 1. The fence is homeomorphic to a solid torus.

Now we are in the position to investigate a holonomy about the foliations on the fence induced by a given holomorphic vector field. Let $\hat{F}$ be a foliation on a plumbed 4-manifold represented by a final resolution picture of a given vector field and construct a flow which is tangent to the leaves of $\hat{F}$ with a saddle structure at this singularity as in $\S 3$ ([4]). By using this fact a real one dimensional flow (or a foliation) induced by 


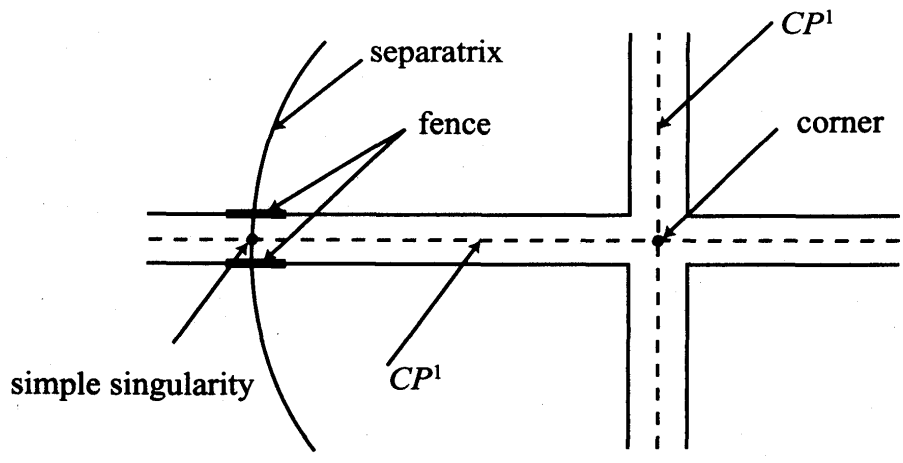

Figure 1

the foliation $\hat{F}$ is defined on this fence (see [4]). The real foliations are defined only in a neighborhood of each non-zero simple singularities of $\hat{F}$. Thus we reduce the study of the holonomy of the above foliations to the study of the holonomy of foliations on the same fence defined in a neighborhood of the singularity $o$ of the differential equation $d z / d T=Z(z), z=\left(z_{1}, z_{2}\right), D Z(o)=\left(\begin{array}{cc}\lambda_{1} & 0 \\ 0 & \lambda_{2}\end{array}\right)$ with resonance such that $m_{1} \lambda_{1}+m_{2} \lambda_{2}=0$, where $o$ is the origin of $\mathbf{C}^{2}$ and $m_{1}$ and $m_{2}$ are positive integers. Camacho and Sad ([1] and [4]) studied the holonomy of foliations on the fence near the origin $o$ in $\mathbf{C}^{2}$. A holonomy map induced by a flow (or a foliation) on a tubular neighborhood of a certain circle (or a solid torus) is written as follows:

LEMMA 1 ([2]). Consider a holomorphic ordinary differential equation $d z / d T=Z(z)$ such that $z=\left(z_{1}, z_{2}\right)$ and $D Z(o)=\left(\begin{array}{cc}\lambda_{1} & 0 \\ 0 & \lambda_{2}\end{array}\right)$ with resonance $m_{1} \lambda_{1}+m_{2} \lambda_{2}=0\left(m_{1}, m_{2} \in \mathbf{N}\right)$. Let $F_{Z}$ be a foliation induced by the above equation on a neighborhood of the origin, and let $\gamma$ be a small circle of which center is the origin in $\mathbf{C}^{2}$ defined on the plane $z_{1}=0$. Let $X$ denote a one dimensional real foliation induced by $F_{Z}$ as above and $f$ denote a holonomy map with respect to the foliation $X$ on the fence of $\gamma$. Then the map $f$ is written as follows:

$$
f(z)=\mu_{1} z+A z^{k n+1}+\cdots, \quad \text { where } \mu_{1}=\exp 2 \pi\left(\lambda_{1} / \lambda_{2}\right) i .
$$

Similarly, take a small circle $\gamma^{\prime}$ on the plane $z_{2}=0$, and denote a holonomy map on the fence of $\gamma^{\prime}$ by $g$. Then the map $g$ is written as follows:

$$
g(z)=\mu_{2} z+B z^{k m+1}+\cdots \quad \text { where } \mu_{2}=\exp 2 \pi\left(\lambda_{2} / \lambda_{1}\right) i,
$$

and integers $m, k$, and $n$ are given by the indices of the following normal form of $d z / d T=$ $Z(z)$;

$$
\frac{d z_{1}}{d T}=\lambda_{1} z_{1}+a z_{1}^{k m+1} z_{2}^{k n}+R_{1}
$$




$$
\frac{d z_{2}}{d T}=\lambda_{2} z_{2}+b z_{1}^{k m} z_{2}^{k n+1}+R_{2}
$$

The map $f$ in Lemma 1 is topologically conjugate to one of the following normal forms:

$$
f_{\text {normal }}(z)=\lambda z\left(1+z^{k m_{1}}\right) \text { or } f_{\text {normal }}(z)=\lambda z,
$$

where $\lambda=\exp \left(-m_{2} / m_{1}\right) 2 \pi i$ (see Theorem 1 in [1]). Let us consider the dynamics of the map $f_{\text {normal }}$. $\bigcap\left(f_{\text {normal }}^{m_{1}}\right)^{p}\left(D^{2}\right)-\{o\}$ is divided into $2 k m_{1}$ connected components as in Figure 2. Let $z_{0}$ be a point contained in some component of $\bigcap\left(f_{\text {normal }}^{m_{1}}\right)^{p}\left(D^{2}\right)-\{o\}$.

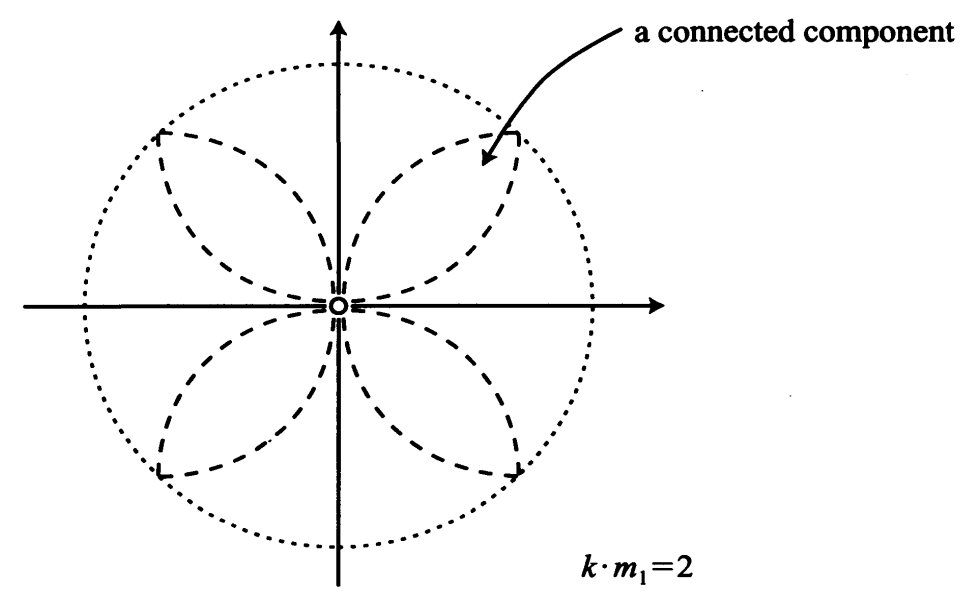

FIGURE 2

Then the component containing the point $f_{\text {normal }}^{i}\left(z_{0}\right)(i=0,1,2,3, \cdots)$ coincides with the component containing the point $L f_{\text {normal }}^{i}\left(z_{0}\right)(i=0,1,2,3, \cdots)$, where $L f_{\text {normal }}$ denotes the linear part of the map $f_{\text {normal }}$. Since the map $L f_{\text {normal }}$ is a periodic map with the period $m_{1}$, the point $f_{\text {normal }}^{m_{1}}\left(z_{0}\right)$ and the point $z_{0}$ are contained in the same component. We can join successively the points $z_{0}, f_{\text {normal }}\left(z_{0}\right), f_{\text {normal }}^{2}\left(z_{0}\right), \cdots$, and $f_{\text {normal }}^{m_{1}}\left(z_{0}\right)$ by using the orbits of the flow $X$. If the point $z_{0}$ does not coincide with the point $f_{\text {normal }}^{m_{1}}\left(z_{0}\right)$, join $z_{0}$ and $f_{\text {normal }}^{m_{1}}\left(z_{0}\right)$ by a certain arc contained in the above component. Then we get a closed orbit around $\gamma$ or a circle approximating an orbit of $X$ passing through $z_{0}$ in the neighborhood of $\gamma$. Both circles are $\left(m_{1}, m_{2}\right)$-cable around $\gamma$. If the circle is an approximating orbit of $X$ and does not coincide with any orbits of $X$ in the tubular neighborhood of $\gamma$, we call this circle a pseudo $\left(m_{1}, m_{2}\right)$-cable orbit around $\gamma$.

Now let $N$ be a neighborhood of the origin in $\mathbf{C}^{2}$ and $\hat{U}$ be a plumbed 4-manifold represented by a final resolution picture of a given vector field. $\hat{F}$ denotes a foliation on $\hat{U}$ induced by the desingularized vector field. The foliation $\hat{F}$ on $\hat{U}-\bigcup C P^{1}$ is topologically equivalent to the foliation $F$ induced by a given vector field defined on $U-\{o\}$. There is a fence on a small circle in a leaf of $\hat{F}$ in $\hat{U}$ of which the center is a non-zero simple singularity. Here, the leaf which has a small circle defined as above 
becomes a separatrix in $U$ by the blowing-down operations. We denote by $X_{\partial U}$ the foliation on the boundary of $U$ induced by the given holomorphic vector field $Z$ on $U$ in $\mathbf{C}^{2}$. Every component of the separatrices transversely intersects with the boundary of $U$ at a circle. If we choose the separatrix in $U$ which becomes the leaf of $\hat{F}$ in $\hat{U}$ containing the above circle by blowing-up, then we can define the tubular neighborhood $N$ of the intersection constructed of this separatrix in the boundary of $U$. We restrict the foliation $X_{\partial U}$ to this neighborhood $N$ and denote it by $X_{\partial U} \mid N$. Then the foliation on the neighborhood of the circle near the simple singularity in $\hat{U}$ is topologically equivalent to the foliation $X_{\partial U} \mid N$ since the foliation $\hat{F}$ of $\hat{U}-\bigcup C P^{1}$ is topologically equivalent to the foliation $F$ on $U-\{o\}$. Thus the holonomy map of the foliation on the fence in $\hat{U}$ is also topologically conjugate to the holonomy map of the foliation $X_{\partial U} \mid N$ on a certain small disk which corresponds to the fence in $U$ under the leaf preserving homeomorphism between $U-\{o\}$ and $\hat{U}-\bigcup C P^{1}$. The next two lemmas assert that the multiplicity defined in Definition 3 is invariant under the leaf preserving homeomorphism.

Lemma 2. Suppose that vector fields $Z$ and $Z^{\prime}$ are defined on a neighborhood of the origin $o \in \mathbf{C}^{2}$ and that there exists a topological equivalence between $Z$ and $Z^{\prime}$ near the origin $o \in \mathbf{C}^{2}$. Moreover suppose that the orbits induced by $Z$ on the neighborhood $N_{Z}$ of the circle $\gamma_{z}$ are $\left(m_{2}, \pm m_{1}\right)$-cables of $\gamma_{Z}$ and the orbits induced by $Z^{\prime}$ of the corresponding circle $\gamma_{Z^{\prime}}$ are $\left(m_{2}^{\prime}, m_{1}^{\prime}\right)$-cables of $\gamma_{Z^{\prime}}$. Then $m_{2}=m_{2}^{\prime}$.

Here the circle $\gamma_{z}$ is the intersection between the boundary of the neighborhood $U_{Z}$ of the origin $o$ and a certain separatrix of $Z$ and this separatrix becomes a curve through the simple singularity in the plumbed 4-manifold $\hat{U}_{Z}$ with the resonance condition $m_{1} \lambda_{1}+m_{2} \lambda_{2}=0$ by performing the desingularization of $Z$, where $\lambda_{1}$ and $\lambda_{2}$ are the eigenvalues of this simple singularity in $\hat{U}_{z}$. We assume that the circle $\gamma_{z}$ corresponds to $\gamma_{Z}$, by the leaf preserving homeomorphism which defines the topological equivalence between $Z$ and $Z^{\prime}$.

Also we attend to the fact that the integer $\left|m_{1}\right|$ may not be invariant under the leaf preserving homeomorphism, because it may not be extended to the divisor when we perform blowing-up. The number $m_{2}$ (resp. $m_{2}$ ) defines the winding number of the orbit of $Z$ (resp. $Z^{\prime}$ ) around the circle $\gamma_{Z}$ (resp. $\gamma_{Z^{\prime}}$ ).

Proof. Since $Z$ and $Z^{\prime}$ have a topological equivalence there exists a map defined on the neighborhood $U_{Z}$ of the origin $o \in \mathbf{C}^{2}$ which sends every leaf of the foliation $F_{Z}$ induced by $Z$ to a leaf of the foliation $F_{Z^{\prime}}$ induced by $Z^{\prime}$. We denote this map by $h$. The neighborhood $U_{Z^{\prime}}$ of the origin $o \in \mathbf{C}^{2}$ denotes the image of $U_{Z}$ under the homeomorphism $h$. Suppose that the plumbed 4-manifold $\hat{U}_{Z^{\prime}}$ is induced by $U_{Z^{\prime}}$ as the results of a desingularization of the vector field $Z^{\prime}$. Notice that the map $h$ induces the leaf preserving homeomorphism $\hat{h}: \hat{U}_{Z}-\bigcup C P^{1} \rightarrow \hat{U}_{Z^{\prime}}-\bigcup C P^{1}$. This map $\hat{h}$ defines a topological equivalence between the foliation defined on $\hat{U}_{Z}$ around the neighborhood 
of its non-zero simple singularity and the foliation on $\hat{U}_{Z^{\prime}}$ around the neighborhood of the corresponding simple singularity. So the leaf which passes through this singularity in $\hat{U}_{z}$ and is not contained in the divisor is sent to a leaf which passes through the corresponding singularity in $\hat{U}_{Z}$.

Let $g_{1}(x, y)$ and $g_{2}(x, y)$ be holomorphic functions having degree greater than two and defined on the neighborhood of $o \in \mathbf{C}^{2}$. Consider the equations:

$$
\begin{aligned}
& \dot{x}=\lambda_{1} x+g_{1}(x, y) \\
& \dot{y}=\lambda_{2} y+g_{2}(x, y)
\end{aligned}
$$

where the eigenvalues $\lambda_{1}$ and $\lambda_{2}$ satisfy the resonance condition $m_{1} \lambda_{1}+m_{2} \lambda_{2}=0$. We assume that there is a simple singularity of the foliation defined by the equation $(*)$ on a neighborhood in $\hat{U}_{z}$. We denote this neighborhood by $V$. So the leaf represented by the equation $y=0$ in a local coordinate $(x, y)$ about this simple singularity $(0,0)$ in $V$ corresponds to the given separatrix in $\mathbf{C}^{2}$ as the results of blow-down operations. Suppose that the separatrix in $\mathbf{C}^{2}$ intersects with the circle $\gamma_{Z}$ in a small $S^{3}$ around the origin $o \in C^{2}$. Take a small circle $\gamma$ on the leaf defined by the equation $y=0$ in the neighborhood of the simple singularity of which the center is the singularity and let $f_{Z}$ (resp. $f_{Z^{\prime}}$ ) be the holonomy map on a small disk $D$ (resp. $D^{\prime}$ ) which intersects with $\gamma$ (resp. $\left.\gamma^{\prime}\right)$ transversely in a neighborhood $\hat{N}_{\gamma}$, (resp. $\left.\hat{N}_{\gamma^{\prime}}\right)$ of $\gamma\left(\right.$ resp. $\left.\gamma^{\prime}\right)$. We assume that the disk $D^{\prime}$ is the image of $D$ under the map $\hat{h}: \hat{U}_{Z}-\bigcup C P^{1} \rightarrow \hat{U}_{Z^{\prime}}-\bigcup C P^{1}$. We write $\hat{N}_{\gamma^{\prime}}=\hat{h}\left(\hat{N}_{\gamma}\right)$. Then $f_{Z}$ and $f_{Z^{\prime}}$ are topologically conjugate, i.e., $s f_{Z^{\prime}}{ }^{-1}=f_{Z^{\prime}}$ for some homeomorphism $s:(\mathrm{C}, 0) \rightarrow(\mathrm{C}, 0)$. Every orbit in $N_{Z}$ induced by the vector field $Z$ is a cable knot $\gamma_{Z}$ in $S^{3}$. Thus we can assume that $f_{Z}$ is defined by the normal form $f_{Z_{\text {normal }}}(u)=\exp \left(-2 \pi i m_{1} / m_{2}\right) u([1])$. It is easily checked that the simple singularity with resonance condition $m_{1} \lambda_{1}+m_{2} \lambda_{2}=0$ corresponds to a non-zero simple singularity with a certain resonance condition. So every orbit around the circle $\gamma^{\prime}$ in $\hat{N}_{\gamma^{\prime}}$ defines a cable knot of $\gamma^{\prime}$. Notice that $\hat{U}_{Z^{\prime}}-\bigcup C P^{1}$ is homeomorphic to $U_{Z^{\prime}}-\{o\}$, and there exists a homeomorphism $k^{\prime}: \hat{U}_{Z^{\prime}}-\bigcup C P^{1} \rightarrow U_{Z^{\prime}}-\{o\}$ which sends the leaves of the foliation of $\hat{U}_{Z^{\prime}}-\bigcup C P^{1}$ to the leaves of the foliation of $U_{Z^{\prime}}-\{o\}$. The map $k^{\prime}$ sends $\gamma^{\prime}$ to $\gamma_{Z^{\prime}}$. So we see that every orbits around the circle $\gamma^{\prime}$ in $\hat{N}_{\gamma^{\prime}}$ defines the cable knot of $\gamma_{Z^{\prime}}$ in $N_{Z^{\prime}}$. Suppose that $\hat{N}_{\gamma^{\prime}}=k^{\prime-1}\left(N_{Z^{\prime}}\right)=k^{\prime-1} h\left(N_{Z}\right)$ (see the commutative diagram below).

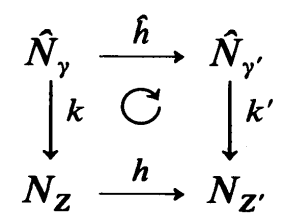

Using Theorem 1 in [1] and Lemma 2, we can define the normal form $f_{Z^{\prime} \text { normal }}$ of $f_{Z^{\prime}}$ by $f_{Z^{\prime}{ }_{\text {normal }}}(u)=\exp \left(-2 \pi i m_{1}^{\prime} / m_{2}^{\prime}\right) u$, since every orbit around the circle $\gamma_{Z^{\prime}}$ in $N_{Z^{\prime}}$ is $\left(m_{2}^{\prime}, m_{1}^{\prime}\right)$-cable knot of $\gamma_{Z^{\prime}}$. Hence we have that $s f_{Z}^{m_{2}} s^{-1}(u)=f_{Z^{\prime}}^{m_{2}}(u)=\exp \left(-2 \pi i m_{1}^{\prime} / m_{2}^{\prime}\right)$ $\cdot m_{2} u$. The point $u$ agrees with $f_{Z_{\text {normal }}}^{m_{2}}(u)$. Thus we obtain $m_{2} / m_{2}^{\prime} \in \mathbf{Z}$. We also obtain $m_{2}^{\prime} / m_{2} \in \mathbf{Z}$ from the fact $s^{-1} f_{Z_{\text {normal }}^{\prime \prime 2}}^{m_{2}^{\prime}}(u)=f_{Z_{\text {normal }}}^{m_{2}^{\prime}}(u)$. Hence we obtain $m_{2}=m_{2}^{\prime}$. 
LEMMA 3. Suppose that the circle $\gamma_{Z}$ and $\gamma_{Z^{\prime}}$ are the same as in Lemma 2 and that every orbit induced by $Z$ in the neighborhood $N_{Z}$ of the circle $\gamma_{Z}$ is pseudo $\left(m_{2}, m_{1}\right)$-cable orbit around $\gamma_{2}$. Then the orbits induced by $Z^{\prime}$ in the tubular neighborhood $N_{Z^{\prime}}$ corresponding to the ones of $Z$ by the leaf preserving homeomorphism are also certain pseudo cable orbits. If the type of them are defined by the pair of integers $\left(m_{2}^{\prime}, m_{1}^{\prime}\right)$, then $m_{2}^{\prime}=m_{2}$.

Proof. Firstly, we must remark that pseudo orbits in $N_{Z}$ are not sent to any pseudo orbits in $N_{Z}$, by leaf preserving homeomorphism as in the proof of Lemma 2 . But we can define the holonomy map $f_{Z}$ (resp. $f_{Z^{\prime}}$ ) on the small disk $D$ (resp. $D^{\prime}$ ) which transversely intersects with the circle $\gamma_{Z}\left(\right.$ resp. $\gamma_{Z^{\prime}}$ ) as in Lemma 2. Also we see that $f_{Z}$ and $f_{Z^{\prime}}$ are topologically conjugate. The pseudo cable orbit in $N_{Z}$ is defined by a pair of integers $\left(m_{2}, m_{1}\right)$. Hence by Theorem 1 in [1], the holonomy map $f_{Z}$ has the normal form:

$$
f_{Z_{\text {normal }}}(u)=\exp \left(-2 \pi i m_{1} / m_{2}\right) u+c_{1} u^{k m_{2}+1}+\cdots .
$$

Since every orbit around $\gamma_{Z^{\prime}}$ in $N_{Z^{\prime}}$ induced by the vector field $Z^{\prime}$ is not closed, we can construct a pseudo cable orbit around $\gamma_{Z}$, by using the same arguments as stated before Lemma 2. Take the normal form of the holonomy map of $f_{Z^{\prime}}$;

$$
f_{Z^{\prime}{ }_{\text {normal }}}(u)=\exp \left(-2 \pi i m_{1}^{\prime} / m_{2}^{\prime}\right) u+c_{2} u^{k m_{2}^{\prime}+1}+\cdots
$$

and let the map $s: D \rightarrow D^{\prime}$ be the same as defined in the proof of Lemma 2. Then

$$
s f_{Z_{\text {normal }}}^{m_{2}} s^{-1}(u)=f_{Z_{\text {normal }}}^{m_{2}}(u)=\exp \left(-2 \pi i m_{1}^{\prime} / m_{2}^{\prime}\right) m_{2} u+\cdots
$$

since $f_{Z}$ and $f_{Z^{\prime}}$ are topologically conjugate. Here the point $u$ and $f_{Z_{\text {normal }}}^{m_{2}}(u)$ are contained in the same connected component which is invariant under $f_{Z_{\text {normal }}}^{m_{2}}(u)$ (cf. [1]). Thus we obtain $m_{2} / m_{2}^{\prime} \in \mathbf{Z}$ and also $m_{2}^{\prime} / m_{2} \in \mathbf{Z}$ from the fact $s^{-1} f_{Z_{\text {normal }}^{\prime \prime}}^{m^{\prime}} s(u)=f_{Z_{\text {normal }}}^{m_{2}^{\prime}}(u)$. This implies that $m_{2}=m_{2}^{\prime}$. Take a holomorphic vector field defined on a certain neighborhood $U$ of the origin $o \in \mathbf{C}^{2}$ as above. There is a foliation around its non-zero simple singularity on the plumbed manifold $\hat{U}$ which is a final stage of blowing-up operations of the vector field on $U$. Every foliation around its simple singularity is always defined by the differential equations:

$$
\begin{aligned}
& \dot{x}=a x+f_{1}(x, y) \\
& \dot{y}=b y+f_{2}(x, y)
\end{aligned}
$$

choosing a suitable coordinate $(x, y)$ around the singularity in $\hat{U}$ where $a$ and $b$ are eigenvalues of this simple singularity such that $a / b \notin \mathbf{Q}_{+}$and $f_{1}$ and $f_{2}$ are certain holomorphic functions of which degrees are greater than two (see [4]). Here $\mathbf{Q}_{+}=\{x \mid x \in \mathbf{Q}, x \geqq 0\}$. The leaf of the foliation passing through this simple singularity can be defined by the equations $x=0$ and $y=0$ using this coordinate. Now we define the multiplicity of the graph link induced by a holomorphic vector field. 
DEFINITION 3. Suppose $L_{z}$ be a graph link represented by the intersections between a certain small $S^{3}$ around the origin $o \in \mathbf{C}^{2}$ and separatrices of a holomorphic vector field $Z$. Let $\Gamma_{Z}$ be a splice diagram representing the graph link $L_{Z}$, so $\Gamma_{Z}$ has an arrowhead vertex which represents a leaf defined by the equation $\dot{x}=\lambda_{1} x+\cdots, \dot{y}=\lambda_{2} y+\cdots$ which satisfies the resonance condition $m_{1} \lambda_{1}+m_{2} \lambda_{2}=0$. Here we have used a local coordinate $(x, y)$ around the singularity of this foliation on the plumbed manifold. Then we define the multiplicity of a component of the link $L_{z}$ represented by the arrowhead to be $m_{2}$ (resp. $m_{1}$ ).

If an arrowhead vertex of $\Gamma_{Z}$ is induced by a leaf passing through a simple singularity of another type, then the multiplicity is defined to be an integer 1 .

REMARK. It follows from Lemma 2 that the multiplicity in Definition 3 is invariant modulo sign under the leaf preserving homeomorphism between the foliations induced by topologically equivalent vector fields.

Let us call above number $m_{i}(i=1,2)$ or 1 as the vertex weights. Then the Alexander polynomial of $n$-variables $A\left(t_{1}, \cdots, t_{n}\right)$ is defined by the above splice diagram, and the Alexander polynomial of one-variable $A\left(t, t^{-1}\right)$ is induced by the polynomial $A\left(t_{1}, \cdots, t_{n}\right)$ as in Theorem 1 in [8]. Now the polynomial $A\left(t, t^{-1}\right)$ is invariant under an orientation preserving and leaf preserving homeomorphism modulo $\pm t^{N}$ ( $N$ is an integer). By Lemma 2 and Lemma 3, the multiplicity of the arrowhead is invariant under an orientation preserving and leaf preserving homeomorphism up to the sign. Since topological equivalence between given two vector fields induces a graph isomorphism between two splice diagrams obtained from two vector fields, we see that the Alexander polynomial of one-variable is invariant under an orientation preserving and leaf preserving homeomorphism modulo $\pm t^{N}$. Thus we get the next theorem.

THEOREM E. Suppose that $Z$ is a vector field as above and $L_{Z}$ is a multi-link induced by $Z$ and has certain multiplicities as defined in Definition 3. Then the Alexander polynomial of one-variable $A\left(t, t^{-1}\right)$ induced by this multi-link $L_{Z}$ is an invariant under a leaf preserving and orientation preserving homeomorphism modulo $\pm t^{N}$ for some integer $N$.

\section{References}

[1] C. Самасно, On the local structure of conformal mappings and holomorphic vector fields in $\mathbf{C}^{2}$, Astérisque 59-60 (1978), 83-94.

[ 2] C. Camacho, A. L. Neto and P. SAD, Topological invariants and equidesingularization for holomorphic vector fields, J. Differential Geometry 20 (1984), 143-174.

[3] C. САмACHO and P. SAD, Invariant varieties through singularities of holomorphic vector fields, Ann. of Math. 115 (1982), 579-595.

[4] C. CAMACHO and P. SAD, Topological classification and bifurcations of holomorphic flows with resonances in $C^{2}$, Invent. Math. 67 (1982), 447-472.

[5] D. Eisenbud and W. D. Neumann, Three-Dimensional Link Theory and Invariants of Plane Curve Singularities, Ann. Math. Studies 110 (1986), Princeton Univ. Press. 
[6] W. D. Neumann, Complex algebraic plane curves via their links at infinity, Invent. Math. 98 (1989), 445-489.

[7] W. D. Neumann, On the topology of curves in complex surfaces, Toplogical Methods in Algebraic Transformation Groups, Proc. Conf. Rutgers Univ., Progress in Math. 80 (1989), 117-133.

[8] N. OKa, Graph link invariants of isolated singularities of holomorphic vector fields in $\mathbf{C}^{2}$ I, Tokyo J. Math. 19 (1996), 289-316.

Present Address:

Shimotakaido 3-32-7, Suginami-KU, Tokyo, 168 JAPAN. 